\title{
Historia clínica electrónica: factores de resistencia para su uso por parte de los médicos
}

\author{
María Mercedes Chá Ghiglia*
}

\section{Resumen}

La implementación de la historia clínica electrónica (HCE) en las organizaciones de salud constituye un proceso de cambio tecnológico. La sustitución del papel por el registro clínico electrónico (RCE) implica que la historia clínica deja de ser un registro de información con un fuerte componente de propiedad por parte del médico y de la organización, e incorpora la integración de la información, el trabajo en equipo, la multidisciplinariedad, las reglas y normas no propias, el acceso ampliamente distribuido al servicio de diversos fines legales y legítimos.

El objetivo de esta investigación es identificar los factores de resistencia por parte de los médicos para el uso de la HCE en una institución de atención a la salud. Se realizó una investigación cualitativa a través de entrevistas semiestructuradas y se selecciona la muestra con criterio intencional. En el análisis global de las entrevistas se observa la combinación de diferentes factores de resistencia en cada uno de los profesionales, siendo los más reconocidos: la interferencia en la relación médico-paciente, la mayor demanda de tiempo en el inicio, el temor a lo desconocido, la inadecuación del sistema de trabajo, los intereses de los profesionales médicos. Se identifican, asimismo, distintos tipos de usuarios de tecnología: algunos se han apropiado de la misma, otros son usuarios obligados y otros optan por el no uso. Conclusión, algunas de las barreras pueden ser modificadas directamente desde la gestión del proyecto, por ejemplo, la usabilidad del software, o en las consideraciones del factor tiempo. Sin embargo, hay otras que implican cambios vinculados al comportamiento de los profesionales médicos, que suelen estar relacionados con sus valores y sus creencias, razón que hace más compleja la intervención para el cambio.

Palabras clave: Registros electrónicos de salud

Cambio tecnológico

Historia clínica electrónica

Factores de resistencia

Key words: Electronic health records

Technological change

Electronic medical record

Resistance factors

\footnotetext{
* Médico especialista en Nefrología (UDELAR). Especialista en gestión de salud (UDELAR). Directora técnica de CAMOC-IAMPP.

Correo electrónico: mchaghiglia5@gmail.com

La autora declara no tener conflicto de intereses.

Recibido: $29 / 10 / 19$

Aprobado: 5/2/20
} 


\section{Introducción}

La implementación de la historia clínica electrónica (HCE) constituye un proceso de cambio tecnológico. La sustitución del papel por el registro clínico electrónico (RCE) implica que la historia clínica deja de ser un registro de información con un fuerte componente de propiedad por parte del médico y de la organización, e incorpora la integración de la información, el trabajo en equipo, la multidisciplinariedad, las reglas y normas no propias, el acceso ampliamente distribuido, al servicio de diversos fines legales y legítimos ${ }^{(1)}$.

Este cambio de paradigma en cualquier centro médico público o privado se ve acompañado por la aparición de resistencias a su utilización e involucran desde la alta gerencia hasta las áreas administrativas y asistenciales. Las resistencias individuales son planteadas por los individuos de una organización a partir de sus percepciones, personalidades y necesidades ${ }^{(2)}$.

El objetivo del presente estudio fue identificar los factores de resistencia por parte de los médicos para la implementación de la HCE en una organización de atención a la salud.

El Institute of Medicine (IOM, por su sigla en inglés) la define como una colección longitudinal de información electrónica acerca de la salud de las personas, donde la información sobre salud es definida como información pertinente a la salud de un individuo o como la información de los cuidados de salud provistos a un individuo por medio de cualquier miembro del equipo de salud.

\section{La historia clínica electrónica permite:}

- Acceso electrónico inmediato a la información de salud personal o poblacional solamente de usuarios autorizados.

- Provisión de bases de conocimiento y sistemas de soporte para la toma de decisiones que mejoren la calidad, seguridad y eficiencia de la atención de los pacientes.

- Dar soporte efectivo en la eficiencia de los procesos para brindar cuidados de salud ${ }^{(3)}$.

Las funcionalidades de la HCE incluyen el registro clínico electrónico, la gestión de la información, la administración de solicitud y resultados de exámenes complementarios, soporte a los profesionales para la toma de decisiones, soporte a los pacientes, generación de reportes, conectividad y comunicación electrónica.

Durante la implementación de la HCE se crean diferentes subgrupos de acuerdo al uso y apropiación que se realiza de la HCE. En aquellos usuarios que pueden optar, es posible reconocer un grupo de usuarios convencidos por el uso (o no uso), que son quienes se apropiaron de la práctica por la cual se decidieron. Un segundo grupo incluye a los no usuarios obligados, es decir, aquellos que no la utilizan por existir barreras de acceso. Y finalmente, el grupo de usuarios por obligación, que es el que desearía dejar de utilizar esta tecnología. La apropiación tecnológica hace referencia al uso significativo de la tecnología, siendo de menor entidad en los usuarios obligados al uso ${ }^{(4)}$.

\section{Metodología}

\section{Presentación de la organización}

Se realiza un estudio en una organización de salud, prestadora del Sistema Nacional Integrado de Salud (SNIS), perteneciente al sector privado, ubicada en el interior del país, de mediano tamaño de acuerdo a los criterios utilizados por el Ministerio Salud Pública.

Es un prestador que brinda atención en el primer, segundo y tercer nivel. Esta institución cuenta con $500 \mathrm{em}-$ pleados: 26\% médicos (sexo femenino 52\%), 28\% funcionarios de enfermería y $46 \%$ personal de los servicios de apoyo.

En el año 2008, la Federación Médica del Interior decide la creación de un software para el RCE. El alcance incluye registro de historia clínica de emergencia, ambulatoria e internación con la capacidad de interoperar con el sistema administrativo, farmacia y laboratorio. Se inicia la implementación del RCE en emergencia sanatorial en abril de 2015 y luego se expande a los diferentes sectores de la organización. Por razones de funcionamiento institucional, en el momento en que se realiza la investigación en el sector policlínica, conviven la historia clínica en soporte papel y el formato electrónico. En la emergencia e internación sanatorial el registro electrónico es la única opción.

\section{Diseño}

Se opta por una técnica de investigación cualitativa porque se pretende comprender los factores que se encuentran en la base de estas resistencias. Se realiza un muestreo intencional donde el investigador selecciona los informantes que integran la muestra siguiendo un criterio estratégico, "los que por su conocimiento de la situación o del problema a investigar se le antojan ser los más idóneos y representativos de la población a estudiar',(5).

Se diseña una investigación a través de entrevistas en profundidad individual, enfocada y semiestructurada. Esta técnica de investigación cualitativa implica que el entrevistador guía la conversación, pero concede espacio al entrevistado para que exprese sus propios puntos de vista (tabla 1). 
Tabla 1. Instrumento utilizado durante la entrevista.

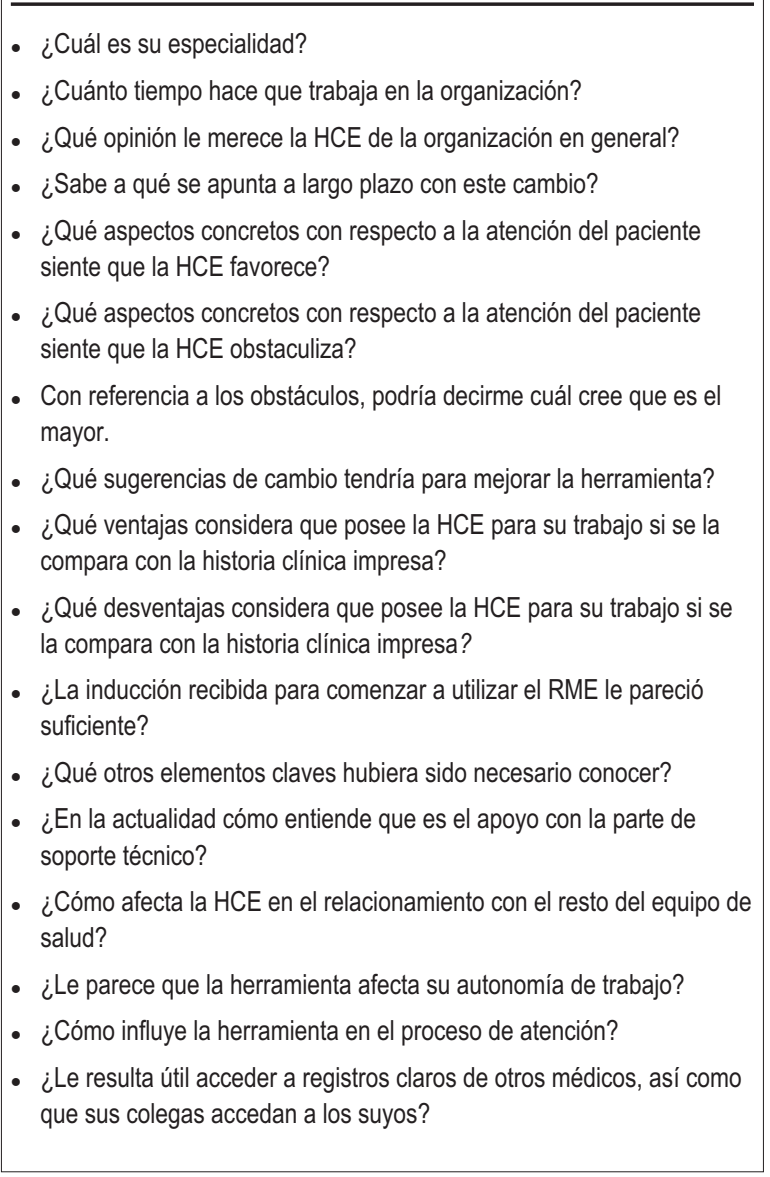

En el período de un mes (mayo 2018), se entrevistaron 16 médicos que ofrecieron resistencia a la utilización de la HCE y requirieron intervención de la dirección técnica para iniciar su uso $(100 \%$ de la población con resistencias). Se incluyen médicos generales, pediatras, especialistas médicos y anestésico-quirúrgicos (tabla 2).

\section{Plan de análisis}

Se identificaron unidades de registro, categorías y temas. El análisis de contenido buscó identificar los aspectos que contribuyen a explicar los factores de resistencia al uso de la HCE que le atribuyen las personas entrevistadas.

\section{Resultados}

En los resultados se observa que el $75 \%$ de la población con resistencia en el uso pertenece al sexo masculino. En su mayoría, los entrevistados reconocen problemas recurrentes tales como la interferencia en la relación médico-paciente, la mayor demanda de tiempo en el inicio, el temor a lo desconocido, la falta de habilidades computacionales y la posibilidad de falla del sistema informático (tabla 3).

La mayoría de los entrevistados se refiere de forma positiva a la capacitación y el apoyo técnico, por lo que consideramos que la falta de entrenamiento, apoyo técnico, limitaciones del sistema, escasez de computadoras e infraestructura no actuaron como barreras en la mayoría de los casos.

\section{Discusión}

El $87,5 \%$ de la población tiene $\geq 40$ años; esto se relaciona con el concepto de brecha digital (noción de que algunos grupos demográficos usan las computadoras e internet con mayor prevalencia que otros). En la literatura se refiere que el uso de la computadora e internet tiene mayor prevalencia entre personas blancas con mayores ingresos y de grupos de edades más jóvenes ${ }^{(5)}$.

En el análisis global de las entrevistas se observa la combinación de los diferentes factores de resistencia en cada uno de los profesionales.

\section{Interferencia en la relación médico-paciente-burocracia}

Un entrevistado menciona: "Desde mi consulta, casi la mitad del tiempo no le miro a la cara al paciente, yo estoy acostumbrado a otra relación en la cual hay idiomas no verbales que yo puedo sacar conclusiones... a veces le digo al usuario, mira que te estoy prestando atención y todo lo que estoy escribiendo está vinculado con lo que tú me estás diciendo" (entrevistado 1). En esta cita se hace mención a la interferencia de la relación médico-paciente entendida como una obstrucción al contacto gestual y visual ${ }^{(6)}$.

Otro entrevistado refiere: "La electrónica le saca tiempo al trato directo con el paciente, si vos hacés preguntas estándar, no lo estás atendiendo..." (entrevistado 15). Acá se manifiesta que la herramienta actúa como burocracia, hallazgo que también es mencionado por Dawidowsky y colaboradores ${ }^{(7)}$. Ninguno de los médicos entrevistados mencionó disconformidades manifiestas por parte de los pacientes, como un obstáculo para su uso.

La interferencia en la relación médico-paciente es mencionado con mucho énfasis por los entrevistados, esto también es referido en la literatura como un cambio en la forma de comunicación tradicional entre los médicos y los pacientes ${ }^{(8)}$.

\section{El factor tiempo}

"Al principio, el obstáculo se presentó en la etapa de aprendizaje, lógicamente ahi hubo un enlentecimiento, eso fue transitorio y eso ya está" (entrevistado 6).

Aquí se considera un obstáculo el aprendizaje en el uso de la herramienta y realizar los registros de forma diferente. En la etapa inicial aumenta la demanda de tiem- 
Tabla 2. Perfil de los médicos entrevistados.

\begin{tabular}{lcc}
\hline Características & $\begin{array}{r}\text { Frecuencia } \\
\text { relativa (\%) }\end{array}$ & $\begin{array}{c}\text { Frecuencia } \\
\text { absoluta }\end{array}$ \\
\hline Sexo & 25 & 4 \\
Mujeres & 75 & 12 \\
Hombres & & \\
\hline Grupos etáreos & 12,5 & 2 \\
$30-40$ & 37,5 & 6 \\
$41-50$ & 31,3 & 5 \\
$51-60$ & 18,7 & 3 \\
$61-65$ & & \\
\hline Distribución por área de desempeño & & 9 \\
Médicas & 56,3 & 7 \\
Quirúrgicas & 43,7 & \\
\hline
\end{tabular}

po y luego disminuye de forma progresiva en la medida en que se automatizó el sistema adquiriendo fluidez, seguramente asociado a la mejora en las habilidades y destrezas del médico. Esto es concordante con otros estudios que indican que la productividad disminuye al principio, pero luego se nivela en el proceso de implementación debido a que se ahorra de otras áreas ${ }^{(7,8)}$.

Se propone para la mejora del proceso de incorporación de la HCE la posibilidad de negociar diferentes tipos de compensaciones (como abonar tiempo extra o disminuir el número de pacientes/hora), para minimizar la sobrecarga de trabajo que conlleva transitoriamente el aprendizaje e incorporación de la herramienta.

Surge como consideración que la herramienta debe facilitar el proceso de trabajo del médico una vez superada la etapa de aprendizaje, y, de ser posible, también durante su desarrollo. Para cumplir con este objetivo es necesario que el equipo responsable del proyecto tenga presente el concepto de usabilidad (vinculado con la facilidad, rapidez y precisión que los usuarios pueden lograr con un set específico), y el de utilidad (que hace referencia a la funcionalidad del sistema) ${ }^{(9)}$. Estos aspectos deben ser contemplados desde la propia creación del software y continuar durante la etapa de implantación y mantenimiento. El trabajo conjunto de informáticos y médicos para mejorar la usabilidad e integrar los componentes de la HCE a una práctica médica fluida se presenta como imprescindible.

\section{Temor a lo desconocido}

De algunas de las entrevistas surgen las siguientes citas:
Tabla 3. Factores de resistencia: percepción negativa con respecto a la HCE, obstáculos.

\begin{tabular}{|c|c|c|}
\hline Categorías analíticas & Subcategorías & $\begin{array}{c}\text { Énfasis en cada } \\
\text { subcategoría(") }\end{array}$ \\
\hline \multirow{2}{*}{$\begin{array}{l}\text { Interferencia en la } \\
\text { relación } \\
\text { médico-paciente. }\end{array}$} & $\begin{array}{l}\text { Obstrucción al contacto } \\
\text { gestual y visual }\end{array}$ & +++ \\
\hline & Burocracia & + \\
\hline Factor tiempo & $\begin{array}{l}\text { Mayor consumo de } \\
\text { tiempo en la etapa inicial }\end{array}$ & +++ \\
\hline \multirow[t]{3}{*}{$\begin{array}{l}\text { Temor a lo } \\
\text { desconocido }\end{array}$} & $\begin{array}{l}\text { Prejuicios acerca de la } \\
\text { demanda de tiempo }\end{array}$ & +++ \\
\hline & $\begin{array}{l}\text { Falta de personalización } \\
\text { del dispositivo }\end{array}$ & +++ \\
\hline & $\begin{array}{l}\text { Pérdida de la privacidad } \\
\text { profesional y personal }\end{array}$ & + \\
\hline $\begin{array}{l}\text { Desconfianza hacia la } \\
\text { herramienta } \\
\text { informática }\end{array}$ & $\begin{array}{l}\text { Pérdida de la información } \\
\text { durante el proceso de } \\
\text { implantación y la } \\
\text { integración de los } \\
\text { sistemas }\end{array}$ & ++ \\
\hline $\begin{array}{l}\text { Inadecuación al } \\
\text { sistema de trabajo de } \\
\text { los profesionales } \\
\text { médicos }\end{array}$ & Servidor de terminología & +++ \\
\hline $\begin{array}{l}\text { Intereses de los } \\
\text { profesionales } \\
\text { médicos }\end{array}$ & $\begin{array}{l}\text { Interés en algún los de } \\
\text { los componentes de la } \\
\text { HCE, según la } \\
\text { especialidad. }\end{array}$ & +++ \\
\hline \multicolumn{3}{|c|}{$\begin{array}{l}\text { * Las cruces esquematizan el énfasis en cada categoría. } \\
+(\leq 4 \text { veces }) \\
++(4-8 \text { veces }) \\
+++(\geq 8 \text { veces })\end{array}$} \\
\hline
\end{tabular}

"Los pacientes pueden leer claramente... pueden entender y pueden ver y saber lo que uno ha escrito sobre ellos. Pro y contra, pro porque entienden, en contra porque a veces malinterpretan lo que uno escribe" (entrevistado 5).

"Lo más importante es que yo tenía miedo a que la historia clínica electrónica no fuera amena... para usarla en los tiempos que tenemos marcada la consulta. Ahora, a mí, me lleva menos tiempo que la de papel" (entrevistado 10).

"Miedo ancestral, digamos, de lo nuevo... El temor de equivocarme o de que de repente tenía ahi y se te borraba todo lo que había hecho otro incluso... Escribir hace 60 años que sé, computación no" (entrevistado 11).

Las citas anteriores manifiestan el temor a lo desconocido por los diferentes usuarios. En el primer caso, se 
hace referencia a un área de conflicto particular que refiere a la pérdida a la privacidad personal y profesional. El acceso ampliamente distribuido que tiene la HCE se asocia a un cambio de paradigma en la atención en salud que consiste en un empoderamiento de los ciudadanos con mayor accesibilidad a la información sobre su salud. La mayor disponibilidad de información en usuarios, que tal vez no tengan los conocimientos necesarios para leer estos datos, puede repercutir en la relación médico-paciente o paciente-organización.

En el segundo y tercer caso se hace referencia a determinados prejuicios acerca de la demanda de tiempo y la posible falta de personalización del dispositivo para atender necesidades específicas del usuario.

También operan las escasas habilidades computacionales, vinculadas a dificultad para el manejo de los dispositivos (digitalización, manejo básico de informática, entre otros); en algunos casos se manifiestan como una dificultad generacional, esta brecha es superada por algunos médicos. Surgen expresiones de proceso adaptativo como la siguiente: "No soy un gran internauta, pero aprendí, aprendí a la fuerza ... choqué con todo y aprendi" (entrevistado 13). En este sentido, adquiere relevancia la disposición para aprender, parece existir consenso en cuanto a que el aprendizaje es "un proceso de mejora personal, resultado de todas las experiencias que la persona encuentra en su progresión y que solo existe si se refleja en la acción, es decir, en las reglas de decisión del individuo. El aprendizaje puede ser realizado en lugares formales o informales y es propio del individuo"(10). Entonces, existen factores personales que inciden en la decisión de aprender o no aprender que podrían ser las causas de las resistencias individuales, expresiones como: "Lo que capaz que uno puede mejorar, yo y todos, que no depende tanto o solo de la HCE, sino del esfuerzo que cada uno de nosotros haga o que logremos entender el valor que tiene" (entrevistado 4).

\section{Desconfianza hacia la herramienta informática}

"Nosotros, cada vez que vamos y prendemos la computadora... no sabemos si va a funcionar o no va a funcionar, si el programa va a estar o no, si va a estar modificado, entonces la forma de implementación también es importante... lo ideal es que en este proceso no se pierda información que pueda ser valiosa". Se vincula el temor a la pérdida de información durante el proceso de implantación del nuevo sistema y de configuración con los sistemas existentes, como así también las eventuales fallas en el respaldo energético o informático.

El siguiente fragmento "justamente que se cae el sistema, propiamente el PACS (Picture Archiving and Comunications System)", que es un sistema de almacenamiento y transmisión de imágenes, "eso a veces veo que pone un poco de mal humor al paciente, por el tiempo, hay gente que viene con tiempo y otra que no" (entrevistado 3), hace referencia a la dificultad de acceso a la información y al software en un entorno de restricciones de tiempo.

\section{Inadecuación del sistema de trabajo de los profesionales} médicos

"Querer poner el diagnóstico correcto como nosotros lo manejamos en los libros y como lo tenemos protocolizado" (entrevistado 14). Esta cita manifiesta la dificultad en el uso de la terminología que desarrollaremos posteriormente; otro, considera que afecta además la autonomía en su trabajo: "Es una cosa que me tiene aburrido... esta historia, el idioma de esta historia no corresponde al Uruguay... dejo que pase al comité de consultas" (entrevistado 12).

La dificultad en el uso del servidor de terminología es referida como unos de los elementos de fracasos en la implementación de estos sistemas. En la interfaz del registro médico electrónico existen campos estructurados y la codificación que obliga a los profesionales a sistematizar algunos campos, como motivo de consulta y diagnósticos clínicos a través del servidor de terminología. Esto último implica un cambio con respecto al lenguaje narrativo que utilizan los médicos.

De esta cita, se desprende además que el profesional entrevistado está utilizando la herramienta, sin apropiarse de la tecnología, es decir, sin integrarla a sus prácticas habituales. La práctica de uso descrita tiene aspectos positivos, contribuyendo a incorporar términos de uso regional al servidor de terminología y, lo negativo, que no sabemos hasta dónde se maximiza el uso de la herramienta actual. Uno de los desafíos más grandes que tiene la informática médica es tener un vocabulario que represente el conocimiento clínico y que pueda utilizarse en sistemas informáticos. A través de los datos estructurados las cosas que pasan en el mundo real pueden ser entendidas por la computadora.

La carga de datos estructurados para los usuarios del sistema de salud es un obstáculo en la adopción de la $\mathrm{HCE}$, dado que los profesionales prefieren documentar los hallazgos, los procesos y los resultados de la atención médica en texto libre, sin restricciones. El lenguaje natural es enorme, muy detallado, pero ambiguo y con dependencia del contexto. La entrada de datos de forma estructurada no admite la flexibilidad y la expresividad a la que los médicos están acostumbrados, en estos casos es difícil interpretar y reconstruir el significado si hay pérdida de información contextual ${ }^{(6)}$. 


\section{Intereses de los profesionales médicos}

"Yo creo que en los últimos 10 a 15 años los médicos somos menos autónomos y cada vez trabajamos más en equipo, tenemos que tener una visión más global del paciente" (entrevistado 10). En el mismo sentido, otro entrevistado menciona "hay algunos que te dan toda la información para decidir futuras conductas... Y hay otros que no ponen nada" (entrevistado 7).

Un entrevistado utiliza un componente de HCE y realiza el siguiente comentario "me encantó, espectacular la herramienta para medir angulaciones de columna, de escoliosis... y no depender del paciente que si trajo la placa... tener toda la información ahí para nosotros fue sensacional... una herramienta muy útil".

Una de las funcionalidades de la HCE es facilitar la accesibilidad a la información, las especialidades quirúrgicas priorizan el acceso al componente de imágenes; sin embargo, otros jerarquizan el registro médico propio y de colegas. El tipo de información requerida por los médicos es variable de acuerdo con la especialidad que desempeña, algunas especialidades realizan un abordaje integral del paciente con equipos multidisciplinarios y perciben de forma muy positiva el registro de otros colegas. En este grupo, la falta de registro por parte de determinados colegas es identificada como un obstáculo para la atención del paciente.

Esto también pone en evidencia la importancia de articular los diferentes componentes de la $\mathrm{HCE}$, centrados en el usuario, de manera que toda la información sobre el paciente esté disponible. No obstante, hay una parte de usuarios obligados al uso de la tecnología que desearían no realizarla, que no considera importante o necesaria esa mirada amplia de la situación de la salud, aplicando una práctica de modelo fragmentada.

Otro aspecto de análisis es el rol que puede tener el incentivo económico o las penalizaciones sobre el registro médico. La siguiente cita vincula el registro en la HCE con el pago del acto médico (entendido como el pago cuya unidad de servicio es el acto médico), se menciona: "Lo bueno de este sistema es que es un sistema que lo veo acá y en otra organización... Además, no haces la historia, no te facturan el acto..." (entrevistado 15). Este comentario seguramente responde a una política institucional que busca promover el registro electrónico a través de la sanción económica por el no registro, en este caso el no pago del acto asistencial.

Se observa que algunos de los entrevistados que en un inicio mostraron resistencia a su uso, posteriormente pasaron a ser usuarios convencidos y defensores, mostrándose no solo como usuarios de la tecnología, sino también con un alto grado de apropiación. Esta cita es un ejemplo de la situación referida: "Excelente. Esa es mi opinión, es un instrumento que trae múltiples ventajas desde mi punto de vista, registros de mejor calidad en términos de legibilidad, parece un tema menor pero no es un tema nada despreciable.... Obliga a que determinadas cosas queden efectivamente registradas, en cuanto a la HC de papel uno puede pasar por alto determinadas cosas, acá también, pero es más difícil. La posibilidad de interactuar con otros especialistas, con otros médicos, el hecho de poder cuándo se decide el ingreso del paciente, rápidamente establecer la continuidad de asistencia" (entrevistado 6). En este caso, se jerarquiza la utilidad de las funcionalidades de la $\mathrm{HCE}$ como mejor disponibilidad de información, legibilidad, accesibilidad y facilitar la comunicación interna entre los diferentes profesionales.

Sin embargo, algunos otros optaron por no usarla en aquellos sectores donde todavía pueden elegir, siendo, además, usuarios dependientes de la tecnología y con muy poca apropiación de la misma en aquellos lugares donde la HCE es obligatoria. El uso de la HCE no necesariamente implica que la herramienta esté integrada en la práctica médica, lo que queda evidente en algunas de las entrevistas analizadas. Se requiere de la participación activa de sus usuarios para completar el registro y su interés en la información disponible para mejorar la toma de decisiones durante el acto asistencial. En los usuarios convencidos y defensores, participantes activos, parece posible mediante la capacitación y el trabajo en equipo profundizar en la apropiación tecnológica y optimizarla. Sin embargo, el mayor desafío está en lograr la participación activa de los usuarios dependientes de la tecnología, que mantienen un perfil de trabajo más fragmentado y en quienes desde su perspectiva la $\mathrm{HCE}$ no agrega valor a la práctica clínica, excepto en algún componente específico. Este último tipo de atención se presenta coincidente con aquellos usuarios que proponen cambios en la interfaz del registro médico como, por ejemplo, grabaciones de la consulta médica o cambios significativos en la arquitectura del software. Los profesionales que valoran el registro propio lo realizan de forma detallada, lo que sumado a una mayor legibilidad del sistema informático, mejora la calidad de la información a ser visualizada y analizada. Los usuarios obligados manifestaron expresiones de desconfianza y recelo sobre la HCE. Se expresaron negativamente en referencia a medidas de seguridad insuficientes en lo general, y en relación con posibles interpretaciones y exposiciones de anotaciones propias en lo particular. A partir de esta investigación surgen tópicos de interés que quedan planteados, por ejemplo, profundizar en esas desconfianzas con el objetivo de identificar si se corresponden con problemas reales de funcionamiento o son excusas para evitar el recorrido de apropiación al nuevo sistema de registro médico. 
Son interrogantes para futuras investigaciones: ¿cómo se puede lograr la participación activa de estos profesionales dependientes de la tecnología que no tienen interés en las funcionalidades de la herramienta?, ¿existe relación entre la resistencia el uso de la HCE y registros de baja calidad?, ¿qué rol puede tener la consultoría de gestión del cambio en este escenario?

\section{Conclusión}

A partir del análisis, realizamos las siguientes consideraciones:

- La implementación de la HCE es un proceso de cambio tecnológico que se encuentra superpuesto con otros cambios del sistema de salud.

- La incorporación de las TIC en el quehacer de los profesionales médicos es uno de los principales desafíos de este proceso, que conlleva superar barreras para su uso y apropiación.

- El uso de la HCE no implica su apropiación, esto último consiste en el uso significativo de la misma, es decir, su utilización adecuada maximizando los beneficios en la atención del paciente y en el sistema de salud.

- Algunas de las barreras pueden ser modificadas directamente desde la gestión del proyecto (usabilidad del software o el factor tiempo); sin embargo, hay otras que implican cambios vinculados al comportamiento de los profesionales médicos, que suelen estar relacionados con sus valores y sus creencias, razón que hace más compleja la intervención para el cambio.

\section{Summary}

Implementation of electronic medical records (EMRs) in the health system constitutes a process of technological change. Substituting paper for electronic medical records implies the medical record is no longer the registration of information with a great sense of ownership by thy the physician and the institution, and it also brings in the integration of information, team work, multidisciplinariety, rules and norms that are not your own, widely distributed Access to it for different legal and legitimate purposes.

This study aims to identify the factors that may result in doctors being against the use of EMRs in a health care institution. We conducted a qualitative research by means of semi-structured interviews and intentionally defined a sample. The global anslysis of the interviews reveals the combination of different factors for resistance in each one of the professionals, the most well known being: interferences in the doctor-patient relationship, the greater demand of time at the start, fear of the unk- nown, inadequacy of the work system and interests of the health professionals.

Likewise, different kinds of technology users are identified: some have embraced it, others are obliged users and other choose not to use it.

In conclusion, some of the barriers may be directly modified from the project management, as for instance, the possibility of using the software available, or the impact of time. However, there are other barriers that imply changes in the behaviour of medical professionals that are usually connected to their values and beliefs, what makes interventions seeking change more complex.

\section{Resumo}

A implementação do Prontuário Electrónico do Paciente (PEP) nas organizações de saúde constituem um processo de mudança tecnológica. A substituição do papel pelo registro clínico electrónico (RCE) implica que o prontuário do paciente deixe de ser um registro de informação com um forte componente de propriedade por parte do médico e da organização, e incorpora a integração da informação, o trabalho em equipe, a multidisciplinariedade, as regras e normas não próprias, o acesso amplamente distribuído no serviço com diversos fins legais e legítimos.

O objetivo deste trabalho foi identificar os fatores de resistência dos médicos no uso do PEP em uma instituição de saúde. Realizou-se um estudo qualitativo com entrevistas semiestruturadas com seleção de amostra com critério intencional. $\mathrm{Na}$ análise global das entrevistas observou-se a combinação de diferentes fatores de resistência em cada um dos profissionais, sendo os mais reconhecidos: a interferência na relação médico - paciente, uma demanda maior de tempo no início do processo, o temor ao desconhecido, falta de adequação do sistema de trabalho, e interesses dos médicos. Foram identificados também distintos tipos de usuários de tecnologia: alguns a utilizam como própria, outros são usuários obrigados e outros optam por não usar. Conclusão, algumas das barreiras podem ser modificadas diretamente na gestão do projeto como por exemplo, a usabilidade do software, ou nas considerações relacionadas ao fator tempo. No entanto, existem outras que implicam modificações vinculadas ao comportamento dos médicos, que em geral estão relacionados com seus valores e suas crenças, o que faz com que a intervenção para a mudança seja mais complexa.

\section{Bibliografía}

1. López Domínguez O, López Arbeloa P, Temes Montes JL. Los sistemas de información en la gestión de centros asistenciales. En: Temes Montes JL, Mengíbar Torres M, eds. 
Gestión hospitalaria. España: McGraw-Hilles Interamericana, 2013:137-49.

2. Mengíbar Torres M, Del Río Urenda S, Terol Fernández FJ. Gestión del cambio. En: Temes Montes JL, Mengíbar Torres M, eds. Gestión hospitalaria. España: McGraw-Hill Interamericana de España, 2013:501-23.

3. Luna D, Soriano E, González Bernaldo de Quirós F. Historia clínica electrónica. Rev Hosp Ital B Aires 2007; 27(2):77-85.

4. Toboso M. Entre el uso y no uso de la tecnología: un enfoque discursivo de la apropiación tecnológica. Intersticios 2013; 7(2):201-4.

5. Ruiz Olabuénaga JI. El diseño cualitativo. En: Ruiz Olabuénaga JI. Metodología de la investigación cualitativa. $5^{\text {a }}$ ed. Bilbao: Universidad de Deusto, 2012:51-82.

6. Luna D, Otero C, Plazzotta F, Campos F. Sistemas de información para la salud. Buenos Aires: Sociedad Italiana de Beneficencia en Buenos Aires, 2018.

7. Dawidowsky AR, Toselli L, Luna DR, Oberti PF, Soto MA, González Bernaldo de Quirós F. Modificaciones en las perspectivas de los médicos sobre el registro médico electrónico: investigación cualitativa longitudinal. Gac Sanit 2007; 21(5):384-9.

8. Boonstra A, Broekhuis M. Barriers to acceptance of electronic medical records by physicians from systematic review to taxonomy and interventions. BMC Health Serv Res 2010; (10):1-17.

9. Baum A, Luna D, Otero P, Schachner B, Montenegro S, Staccia G, et al. Rediseño de la gestión de la lista de problemas de una historia clínica electrónica utilizando la visión de usuarios. 2006 Disponible en: https://www.academia.edu/ 25281183/Rediseño_de_la_gestión_de_la_lista_de_problemas_de_una_historia_clínica_electrónica_utilizando_la_visión_de_los_usuarios [Consulta: 14 mayo 2019].

10. Centro de Investigación y Documentación sobre problemas de la Economía, el Empleo y las Cualificaciones Profesionales. Valorizar el capital humano. Donostia/San Sebastián: CIDEC, 2000.(Cuadernos de trabajo, 30).

María Mercedes Chá, https://orcid.org/0000-0001-7754-0820 